\title{
Negative Refraction in Möbius Molecules
}

\author{
Y. N. Fang, ${ }^{1,2,3}$ Yao Shen, ${ }^{4}$ Qing Ai, ${ }^{5}$, and C. P. Sun ${ }^{1,3}$, 田 \\ ${ }^{1}$ Beijing Computational Science Research Center, Beijing 100084, China \\ ${ }^{2}$ State Key Laboratory of Theoretical Physics, Institute of Theoretical Physics, \\ Chinese Academy of Sciences, and University of the Chinese Academy of Sciences, Beijing 100190, China \\ ${ }^{3}$ Synergetic Innovation Center of Quantum Information and Quantum Physics, \\ University of Science and Technology of China, Hefei, Anhui 230026, China \\ ${ }^{4}$ School of Criminal Science and Technology, People's Public Security University of China, Beijing 100038, China \\ ${ }^{5}$ Department of Physics, Beijing Normal University, Beijing 100875, China
}

\begin{abstract}
We theoretically show the negative refraction existing in Möbius molecules. The negative refractive index is induced by the non-trivial topology of the molecules. With the Möbius boundary condition, the effective electromagnetic fields felt by the electron in a Möbius ring is spatially inhomogeneous. In this regard, the $D_{N}$ symmetry is broken in Möbius molecules and thus the magnetic response is induced through the effective magnetic field. Our findings open up a new architecture for negative refractive index materials based on the non-trivial topology of Möbius molecules.
\end{abstract}

PACS numbers: 81.05.Xj, 03.65.Vf

\section{INTRODUCTION}

It is recognized that materials with simultaneously negative permittivity and permeability also support the coherent propagation of electromagnetic fields [1], like the usual case where both permittivity and permeability are positive. Due to a reversed phase velocity with respect to the group velocity [2], the boundary condition makes the light to bend in an unexpected direction as if the refractive index appears negative according to the Snell's law. Hence, the effect is termed as negative refraction.

Negative refractive index materials are very promising, e.g., in achieving the electromagnetic field cloaking [3, 4], facilitating the sub-wavelength imaging [5], and crime scene investigation [6]. However, such materials do not naturally exist for the absence of magnetic response at the same frequency as electric response. To overcome this difficulty, delicately designed metamaterials have been proposed and tested. Metamaterials rely on structural designing of the sub-wavelength unit cells to tune the electromagnetic resonant characteristic [4]. A key element commonly involved in such artificial architectures is the configuration of split-ring resonator [ $7-10]$. This structure is analogous to an LC resonator with characteristic frequency $\omega_{0} \sim 1 / \sqrt{L C}$ with $L$ and $C$ being self-inductance and capacitance of the resonator, which is tunable by engineering. A metamaterial with splitring resonators can negatively respond to the magnetic field when $\omega \gtrsim \omega_{0}[9]$. Although the negative refraction has been demonstrated at the visible wavelength, manufacturing of microstructure at the size of $30-100 \mathrm{~nm}$ is required [1]]. Such technically requesting fabrication makes it still an open challenge to scale the materials to 3D bulk.

\footnotetext{
*Electronic address: aiqing@bnu.edu.cn
}

${ }^{\dagger}$ Electronic address: cpsun@csrc.ac.cn
Instead of fabricating those delicately designed structures from the conventional "top-down" approach, an attractive alternative is to generate the dielectric media with self-assembled functional atoms or molecules 13 15]. This "bottom-up" approach is particularly interesting due to the small size of molecules and their associated quantum effects. For example, by utilizing the quantum interference among multi-level atoms, it is possible to suppress the absorption meanwhile keep reasonable optical response [16]. We notice that the molecular ring can not effectively respond to the magnetic field, because there does not exist any split to make the symmetry breaking as shown in Refs. [15, 17] and Appendix A.

In this paper, instead of relying on the split-ring configuration, we describe a potential negative-refracting dielectric medium with the Möbius molecules [18, 19]. Such molecules were theoretically proposed [18] and had been fabricated 20-22] in several experiments. Compared with other molecular-ring-like annulenes, the symmetry of Möbius molecule is lowered by its boundary condition, i.e., the usual $D_{N}$ symmetry is broken here to $C_{2}[19]$. Similar to a superconducting ring with magnetic flux going through [23], this particular boundary condition for Möbius molecule could be canceled by some local unitary transformations. After the transformation, the motion of an electron in the Möbius ring is subject to an effective spatially-inhomogeneous magnetic field, even though the original system is exposed to a physically uniform electromagnetic field [19]. Both electric and magnetic responses can be induced at the same transitions and thus both negative permittivity and permeability can be observed at the same frequencies. In this sense, the negative refraction in Möbius molecules is intrinsically caused by the non-trivial topology of the molecules. Motivated by this discovery, we propose realizing a new kind of metamaterials based on the Möbius molecules.

This paper is organized as follows. In the subsequent section, the Möbius molecule is briefly introduced following Ref. [19]. In Sec. III, the allowed transitions between 
(a)

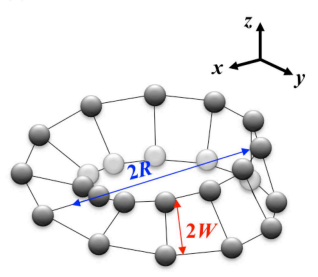

(b) 15

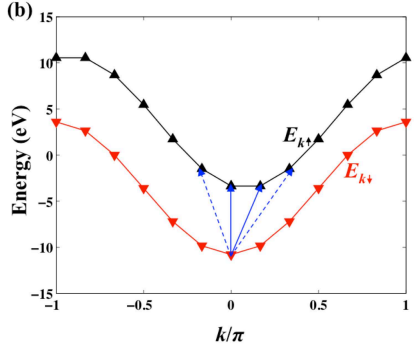

FIG. 1: (color online) (a) Schematic of a molecular ring with Möbius topology: carbon atoms are shown by black circles, with the bonding atoms linked together. (b) Energy spectrum $E_{k \sigma}$ of the molecular ring. Two energy bands are denoted by their different pseudo spin labels $\sigma=\uparrow$ and $\downarrow$. Allowed interband transitions from the ground state are indicated by the colored arrows, where dashed $(x$ and $y)$ and solid $(x, y$, and $z)$ arrows indicate the possible field polarizations.

different eigenstates of a Möbius molecule induced by applied electromagnetic fields are discussed under dipole approximation. Then, in Sec. IV we investigate the possibility of having simultaneously negative permittivity and permeability in a medium consisting of non-interacting Möbius molecules. In Sec. $\mathrm{D}$ the negative refraction is analyzed at a planar medium interface for two different incident conditions. And discussions regarding loss and bandwidth of negative refraction are also given. Finally, the main results are summarized in Sec. VI.

\section{THE MÖBIUS MOLECULAR RING}

The Möbius molecular ring has several physical realizations, e.g., through graphene [24] and the non-conjugated molecules [25]. For a general double-ring system with $2 N$ atoms, as shown in Fig. 1(a), the Hückel Hamiltonian for a single electron reads [19, 26]

$$
H=\sum_{j=0}^{N-1}\left[\mathbf{A}_{j}^{\dagger} \mathbf{M} \mathbf{A}_{j}-\xi\left(\mathbf{A}_{j}^{\dagger} \mathbf{A}_{j+1}+\text { h.c. }\right)\right],
$$

where

$$
\begin{aligned}
& \mathbf{A}_{j}=\left[\begin{array}{l}
a_{j} \\
b_{j}
\end{array}\right], \\
& \mathbf{M}=\left[\begin{array}{cc}
\epsilon & -V \\
-V & -\epsilon
\end{array}\right] .
\end{aligned}
$$

Here, the fermionic operators $a_{j}^{\dagger}\left(b_{j}^{\dagger}\right)$ create a localized atomic-orbital $\left|\phi_{j+}\right\rangle\left(\left|\phi_{j-}\right\rangle\right)$ at the $j$ th nuclear site of the A (B) ring respectively; $2 \epsilon$ describes the on-site energy difference between atoms of two rings; The inter (intra) -ring resonance integral is denoted by $V(\xi)$. Hereafter, we consider a special case where all atoms are of the same species, i.e., $\epsilon=0$ [26], as indicated in Fig. 1(a).

The difference between the Möbius molecular ring and the common chemical annulenes lies in the boundary condition [19]. Here, the $N$ th nucleus in the A ring is exactly the 0th nucleus of the $\mathrm{B}$ ring. Therefore,

$$
\begin{aligned}
& a_{0}=b_{N}, \\
& b_{0}=a_{N}
\end{aligned}
$$

imply that the operators do not obey the periodical boundary condition. Further calculations in Appendix A demonstrate that the vanishing magnetic dipole for the perfect ring results in the absence of negative refraction.

With a local unitary transformation $\mathbf{U}_{j}$ (see Ref. [19] and Appendix (B), the Hamiltonian becomes

$$
H=\sum_{j=0}^{N-1}\left[\mathbf{B}_{j}^{\dagger} V \sigma_{z} \mathbf{B}_{j}-\xi\left(\mathbf{B}_{j}^{\dagger} \mathbf{Q} \mathbf{B}_{j+1}+\text { h.c. }\right)\right],
$$

where

$$
\begin{aligned}
\mathbf{B}_{j} & =\mathbf{U}_{j} \mathbf{A}_{j} \equiv\left[\begin{array}{l}
c_{j \uparrow} \\
c_{j \downarrow}
\end{array}\right], \\
\mathbf{Q} & =\left[\begin{array}{cc}
e^{i \delta / 2} & 0 \\
0 & 1
\end{array}\right], \\
\delta & =2 \pi / N
\end{aligned}
$$

the Pauli matrix $\sigma_{z}$ is written in the pseudo spin space, i.e., $\mathbf{B}_{j}^{\dagger} \sigma_{z} \mathbf{B}_{j}=c_{j \uparrow}^{\dagger} c_{j \uparrow}-c_{j \downarrow}^{\dagger} c_{j \downarrow}$. We emphasize that the new operators $c_{j \sigma}$ 's satisfy the periodical boundary condition. Equation (6) represents a pseudo spin in a fictitious ring [19]. For the spin-up state, the ring is further penetrated by a perpendicular homogeneous magnetic field, as indicated by the phase factor $\exp (i \delta / 2)$ in the hopping term. More insight on the non-trivial boundary condition stems from the corresponding energy spectrum, cf. Fig. 1(b) and Appendix B]

$$
\begin{aligned}
& E_{k \uparrow}=V-2 \xi \cos \left(k-\frac{\delta}{2}\right), \\
& E_{k \downarrow}=-V-2 \xi \cos k,
\end{aligned}
$$

which are obtained by performing the Fourier transform on $\mathbf{B}_{j}$ with the resulting transformed operator denoted as $\mathbf{C}_{k}$ and $k=l \delta(l=0,1, \cdots N-1)$. Compared with the spectrum of a topologically trivial annulene, the effective magnetic flux results in a shift of the $E_{k \uparrow}$ band such that the two lowest states become degenerate. Similar shifting behavior has also been suggested for a cyclic polyene under a real magnetic field [26].

\section{TRANSITION SELECTION RULES}

The general interaction Hamiltonian of a molecule with external electromagnetic field is complicated. But due to the small size of a molecule (typically around a few $\mathrm{nm}$ ) with respect to wavelength the dipole approximation is applicable. In the linear response regime, non-vanishing 
electromagnetic response implies that the corresponding dipole operators have off-diagonal elements between different eigenstates of $H$. Hence it is useful to analyze the transition selection rules under the dipole approximation. We investigate the dipole transition selection rule of the Möbius molecular ring under the perturbation of an external oscillating electric field with amplitude $\vec{E}_{0}$ and frequency $\omega$. The interaction Hamiltonian is written under the dipole approximation as

$$
H_{E}^{\prime}=-\vec{d} \cdot \vec{E}_{0} \cos \omega t
$$

where the electric dipole operator $\vec{d}=-e \vec{r}$ and $\vec{r}$ is the position operator for the electron.

The transition selection rule can be inferred from the matrix elements of $H_{E}^{\prime}$ between the eigenstates of $H$, which are the linear combinations of the atomic orbitals. In the following we ignore the overlap integrals from different atomic orbitals and assume [15, 26]

$$
\left\langle\phi_{j s}|\vec{r}| \phi_{j^{\prime} s^{\prime}}\right\rangle=\delta_{j j^{\prime}} \delta_{s s^{\prime}} \vec{R}_{j s},
$$

where $\vec{R}_{j+}\left(\vec{R}_{j-}\right)$ denotes the position vector of the $j$ th nuclear site at the A(B) ring. For the Möbius molecular ring with radius $R$ and width $4 W$, the nuclear positions are explicitly written in the molecular coordinate system, cf. Fig. 1(a) and Ref. [19], as

$$
\begin{aligned}
\vec{R}_{j \pm}= & \left(R \pm W \sin \frac{\varphi_{j}}{2}\right) \cos \varphi_{j} \hat{e}_{x}+\left(R \pm W \sin \frac{\varphi_{j}}{2}\right) \sin \varphi_{j} \hat{e}_{y} \\
& \pm W \cos \frac{\varphi_{j}}{2} \hat{e}_{z}
\end{aligned}
$$

where

$$
\varphi_{j}=j \delta
$$

is a rotating angle which locates atoms on the rings.

The transition selection rules for the electric dipole operator are briefly summarized as, cf. Appendix C.

$$
\begin{aligned}
|k, \downarrow\rangle & \stackrel{x, y, z}{\rightleftharpoons}|k, \uparrow\rangle,|k, \downarrow\rangle \stackrel{x, y}{\rightleftharpoons}|k+2 \delta, \uparrow\rangle, \\
|k, \sigma\rangle & \stackrel{x, y}{\rightleftharpoons}\left|k \pm \delta, \sigma^{\prime}\right\rangle,|k, \downarrow\rangle \stackrel{z}{\rightleftharpoons}|k+\delta, \uparrow\rangle .
\end{aligned}
$$

Here, $|k, \sigma\rangle$ denotes an eigenstate of $H$ with energy $E_{k \sigma}$. The superscripts $x, y, z$ indicate the electric field polarizations. Those allowed inter-band transitions are schematically shown in Fig. 1(b).

Since the negative refraction depends on the simultaneously negative permittivity and permeability, we further investigate the transition selection rules for the magnetic dipole $\vec{m}$. For molecular systems the current is restricted to the chemical bond, thus $\vec{m}$ is written as [27] $\vec{m}=\sum_{\langle i, j\rangle} J_{i j} \vec{S}_{i j}$, where $\vec{S}_{i j}$ is an effective area specified below. The bond current $J_{i j}$ flows from the $i$ th atom to the $j$ th atom, and by the tight-binding approximation the summation only runs over bonded-atoms pairs. The operator corresponding to the bond current is given by $\hat{J}_{i j}=i e \beta_{i j} a_{i}^{\dagger} a_{j}+$ h.c. [27], where $\beta_{i j}$ is the resonance integral. For the Möbius molecular ring, $\beta_{i j}$ is either $\xi$ or $V$, depending on whether the atoms are on the same ring or not. Consequently, the magnetic dipole operator is explicitly given as

$$
\vec{m}=-e \sum_{j}\left[\mathbf{A}_{j}^{\dagger}\left(V \vec{S}_{j, j}^{+-} \sigma_{y}\right) \mathbf{A}_{j}+\xi\left(i \mathbf{A}_{j}^{\dagger} \overrightarrow{\mathbf{N}}_{j} \mathbf{A}_{j+1}+\text { h.c. }\right)\right]
$$

with the effective area

$$
\vec{S}_{i j}^{\alpha \beta} \equiv \frac{1}{2} \vec{R}_{i \alpha} \times \vec{R}_{j \beta}
$$

$(\alpha, \beta= \pm)$ and the vectorial matrix

$$
\overrightarrow{\mathbf{N}}_{j}=\left[\begin{array}{cc}
\vec{S}_{j, j+1}^{++} & 0 \\
0 & \vec{S}_{j, j+1}^{--}
\end{array}\right] .
$$

Under the dipole approximation, the interaction Hamiltonian describing the coupling of the Möbius molecular ring to an external oscillating magnetic field with amplitude $\vec{B}_{0}$ and frequency $\omega$ is similar to Eq. (12):

$$
H_{B}^{\prime}=-\vec{m} \cdot \vec{B}_{0} \cos \omega t .
$$

The explicit expressions of the matrix elements are complicated in the case of magnetic perturbation and thus we list them in Appendix C] Through straightforward calculations, we find the following transition selection rules as illustrated in Fig. 1(b):

$$
\begin{array}{r}
|k, \downarrow\rangle \stackrel{x y z}{\rightleftharpoons}|k, \uparrow\rangle,|k, \downarrow\rangle \stackrel{x y}{\rightleftharpoons}|k+2 \delta, \uparrow\rangle, \\
|k, \uparrow\rangle \stackrel{x y}{\rightleftharpoons}|k+\delta, \downarrow\rangle,|k, \downarrow\rangle \stackrel{x y z}{\rightleftharpoons}|k+\delta, \uparrow\rangle,
\end{array}
$$

where only the inter-band transitions are shown since in the limit of large $N$, the spectra $E_{k \sigma}$ 's become continuous with respect to $k$ and thus the inter-band transitions are more relevant to the response at high frequency as compared to the intra-band transitions. A comparison between Eq. (21) and Eq. (16) indicates that the selection rules for the inter-band transitions are almost the same for both electric and magnetic couplings.

\section{PERMITTIVITY AND PERMEABILITY}

In order to realize the negative refraction, the simultaneously negative permittivity $\overleftrightarrow{\varepsilon_{r}}$ and permeability $\overleftrightarrow{\mu_{r}}$ are required [1]. To evaluate those quantities, let us consider a negative index medium realized by single crystal of Möbius molecules, with the same orientation for each molecule. Although the permittivity and permeability are originally treated as scalars, for anisotropic medium they could generally be second order tensors that depend on the frequency $\omega$ of external fields $\vec{E}$ and $\vec{H}$. $\overleftrightarrow{\varepsilon_{r}}$ and $\overleftrightarrow{\mu_{r}}$ are calculated by considering that the electric displacement $\vec{D}$ and magnetic induction $\vec{B}$ are related to the 
polarization $\vec{P}$ and the magnetization $\vec{M}$ of the medium [15, 28]:

$$
\begin{aligned}
& \vec{D}=\varepsilon_{0} \overleftrightarrow{\varepsilon_{r}} \vec{E}=\varepsilon_{0} \vec{E}+\vec{P} \\
& \vec{B}=\mu_{0} \overleftrightarrow{\mu_{r}} \vec{H}=\mu_{0} \vec{H}+\mu_{0} \vec{M}
\end{aligned}
$$

Here, $\vec{P}$ and $\vec{M}$ are quantum mechanical averaging of the electric and magnetic dipole operators in the perturbed molecular ground state. In the linear response regime, applying the Green-Kubo formula yields [30], cf. Appendix D.

$$
\begin{aligned}
\vec{P} & =-\frac{1}{\hbar v_{0}} \operatorname{Re} \sum_{k, \sigma}, \frac{\vec{d}_{g, k \sigma}\left(\vec{d}_{k \sigma, g} \cdot \vec{E}\right)}{\omega-\Delta_{k \sigma}+i \gamma}, \\
\vec{M} & =-\frac{\mu_{0}}{\hbar v_{0}} \operatorname{Re} \sum_{k, \sigma}, \frac{\vec{m}_{g, k \sigma}\left(\vec{m}_{k \sigma, g} \cdot \vec{H}\right)}{\omega-\Delta_{k \sigma}+i \gamma} .
\end{aligned}
$$

Here, $\Delta_{k \sigma}=E_{k \sigma}-E_{g}$ is the molecular resonant transition frequency between the excited and ground states of Hamiltonian H. $O_{g, k \sigma}=\langle g|O| k, \sigma\rangle$ is the matrix element of operator $O$ between the unperturbed molecular ground state $|g\rangle$ and excited state $|k, \sigma\rangle$. The ground state is excluded in the summation, as indicated by the prime. The lifetimes $\tau=1 / \gamma$ of the excited states are assumed to be identical. $v_{0}$ is the volume occupied by a Möbius molecule in the medium. In the above calculation, the inter-molecule interactions have been neglected, i.e., the polarization and magnetization are assumed to be the contribution by a collection of non-interacting particles. This approximation is valid either in the low density limit or for medium with crystal structure, e.g., Zinc-Blende [28]. Also, we have verified that both the central frequency and bandwidth of negative refraction are not substantially modified when the inter-molecular interaction is considered at the level of Lorentz local field theory [29] as proven in Appendix $\mathrm{E}$

Because $\overleftrightarrow{\varepsilon_{r}} \simeq 1-\mathrm{O}(|\vec{P}| /|\vec{E}|)$ and $\overleftrightarrow{\mu_{r}} \simeq 1-$ $\mathrm{O}(|\vec{M}| /|\vec{H}|)$, Eqs. (24|25) imply that the negative values of $\overleftrightarrow{\varepsilon_{r}}$ and $\overleftrightarrow{\mu_{r}}$ occur necessarily around the molecular resonant transition frequencies $\Delta_{k \sigma}$. Furthermore, the responses exist only if the corresponding transitions are allowed by both electric and magnetic dipole interactions with fields. As for the Möbius molecular ring, it is particularly interesting that the inter-band transition $|k, \uparrow\rangle \rightleftharpoons\left|k^{\prime}, \downarrow\right\rangle$ could be allowed both electrically and magnetically by the dipole couplings. Besides, the typical value for resonance integral is around the order of a few eV [31, 43], already in the region of visible frequency. Therefore, simultaneously negative permittivity and permeability in the visible frequency regime might be realized in a medium containing molecules with the Möbius topology.

To quantitatively investigate this possibility, $\overleftrightarrow{\varepsilon_{r}}$ is explicitly calculated for the Möbius ring by using the matrix elements $\vec{d}_{k \sigma, g}$. For positive $V$ and $\xi$, the ground state of $H$ is $|0, \downarrow\rangle$ and thus $E_{g}=E_{0 \downarrow}$. When $\omega$ is near the inter-band transition frequency $\Delta_{0 \uparrow}$ and for sufficiently
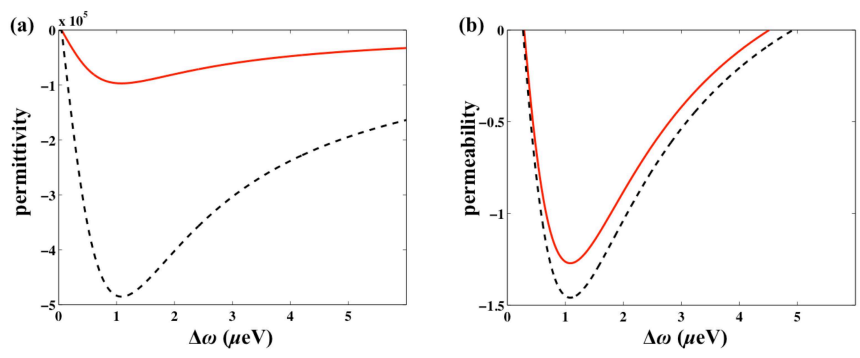

FIG. 2: (color online) Relative permittivity $\overleftrightarrow{\varepsilon_{r}}$ and permeability $\overleftrightarrow{\mu_{r}}$ as a function of the detuning $\Delta \omega=\omega-\Delta_{0, \uparrow}$ between the frequencies of incident field $\omega$ and transition $|0, \downarrow\rangle \rightleftharpoons|0, \uparrow\rangle$. Only negative diagonal elements of $\overleftrightarrow{\varepsilon_{r}}$ and $\overleftrightarrow{\mu_{r}}$ in their corresponding principal-axis coordinate systems are shown. Here we adopt following parameters $V=\xi=3.6 \mathrm{eV}$ [31], $W=0.077 \mathrm{~nm}$ [32], $R=N W / \pi, \gamma^{-1}=4 \mathrm{~ns}$ [39].

long lifetime $\tau, \overleftrightarrow{\varepsilon_{r}}$ is simplified from Eq. (24) by only including the summation term whose denominator contains a transition frequency that is equal to $\Delta_{0 \uparrow}$. With this approximation, the relative permittivity is simplified in the molecular coordinate system as

$$
\overleftrightarrow{\varepsilon_{r}}(\omega)=\left[\begin{array}{ccc}
1-\eta^{\prime}(\omega) & 0 & 0 \\
0 & 1-\eta^{\prime}(\omega) & -2 \eta^{\prime}(\omega) \\
0 & -2 \eta^{\prime}(\omega) & 1-4 \eta^{\prime}(\omega)
\end{array}\right]
$$

where

$$
\eta(\omega)=\frac{1}{8 \hbar \varepsilon_{0} v_{0}} \frac{e^{2} W^{2}}{\omega-2 V-2 \xi\left(1-\cos \frac{\delta}{2}\right)+i \gamma},
$$

and $\eta^{\prime}\left(\eta^{\prime \prime}\right)$ is real (imaginary) part of $\eta$. The permittivity tensor is not diagonal in the molecular coordinate system because of the non-zero off-diagonal term $\varepsilon_{r}^{(y z)}$. Since the tensor is real-symmetric, $\overleftrightarrow{\varepsilon_{r}}$ is diagonalized in the principal-axis coordinate system by a rotation around the $x$ axis. This yields

$$
\varepsilon_{1}=1-5 \eta^{\prime},
$$

$1-\eta^{\prime}$ and 1 for relative permittivity along three principal axes, respectively. In Fig. 2(a), the relative permittivity is numerically demonstrated with the following parameters, $V=\xi=3.6 \mathrm{eV}$ [31], $W=0.077 \mathrm{~nm}$ [32], $\pi R=N W$, $\gamma^{-1}=4$ ns [39], which are obtained by fitting the spectra in experiments.

On the other hand, the tensor of the relative permeability $\overleftrightarrow{\mu_{r}}$ is represented by

$$
\overleftrightarrow{\mu_{r}}(\omega)=\left[\begin{array}{ccc}
1-\alpha^{2} \eta^{\prime} & 0 & 0 \\
0 & 1-\alpha^{2} \eta^{\prime} & -2 \alpha \beta \eta^{\prime} \\
0 & -2 \alpha \beta \eta^{\prime} & 1-4 \beta^{2} \eta^{\prime}
\end{array}\right]
$$

where

$$
\begin{aligned}
\alpha & =\frac{R}{\hbar c}\left[V+\xi\left(\cos \delta-\cos \frac{\delta}{2}\right)\right], \\
\beta & =2 \frac{R \xi}{\hbar c} \sin ^{2} \frac{\delta}{2} \cos \frac{\delta}{2} .
\end{aligned}
$$


The permeability tensor is not diagonal in the molecular coordinate system either. Because $\alpha$ and $\beta$ are generally different from $1, \overleftrightarrow{\mu_{r}}$ and $\overleftrightarrow{\varepsilon_{r}}$ cannot be diagonalized by the same rotating transformation. In other words, the principal axes in which $\overleftrightarrow{\mu_{r}}$ is diagonal do not coincide with the principal axes of $\overleftrightarrow{\varepsilon_{r}}$. This yields

$$
\mu_{1}=1-\left(\alpha^{2}+4 \beta^{2}\right) \eta^{\prime}
$$

$1-\alpha^{2} \eta^{\prime}$, and 1 for relative permeability along three principal axes, cf. Fig. 2(b), respectively.

\section{NEGATIVE REFRACTION AT MEDIUM INTERFACE}

The Möbius medium is anisotropic in the sense that the relative permittivity $\overleftrightarrow{\varepsilon_{r}}$ and permeability $\overleftrightarrow{\mu_{r}}$ are tensors rather than scalars. Therefore, apart from demonstrating simultaneously $\overleftrightarrow{\mu_{r}}<0$ and $\overleftrightarrow{\varepsilon_{r}}<0$ in some frequency region, the more concrete way to show the existence of negative refraction is to investigate the behavior of refracted electromagnetic waves at the medium interface. Here, we investigate the reflection and refraction at a planar interface between the Möbius medium and the air for two specific incident configurations. The results show that the medium is "left-handed" for E-polarized propagating mode [33].

The behavior of a propagating wave inside a medium is captured by its phase and group velocities. For example, when the negative refraction was first introduced in Veselago's seminal paper [1], a "left-handed" material was described as a medium in which the electromagnetic wave propagates with the opposite phase velocity with respect to the group velocity. In media where the permittivity and permeability tensors are symmetric, the group velocity is along the same direction as the Poynting vector 33]. In such case, the reversal of the phase velocity to the Poynting vector has been applied as a criterion for "left-handed" materials: $\vec{k}_{t} \cdot \vec{S}_{t}<0$ [35], where $\vec{k}_{t}$ and $\vec{S}_{t}$ are respectively the wave vector and Poynting vector of refracted field. Besides this, if we consider the refraction from the medium interface, the causality requires that the normal component of its Poynting vector is in the same direction as that for the incident light 36]. In summary, we adopt the following three criteria as characteristics of a "left-handed" medium: (i) The wave vector $\vec{k}_{t}$ in the medium is a real-valued vector; (ii) The wave vector $\vec{k}_{t}$ and Poynting vector $\vec{S}_{t}$ in the medium satisfy $\vec{k}_{t} \cdot \vec{S}_{t}<0$; (iii) The normal component of the Poynting vector remains the same sign across the medium interface, e.g., if interface normal is $\hat{e}_{z}$ then $S_{i z} S_{t z}>0$, where $\vec{S}_{i}$ is the Poynting vector of the incident light.

Let us consider a linearly polarized monochromatic light which is incident from the air onto the interface of Möbius medium as illustrated in Fig. 3. Let $z=0$ be the medium interface and suppose that the incident plane is the $y$ - $z$ plane and $\theta$ is the angle between the incident
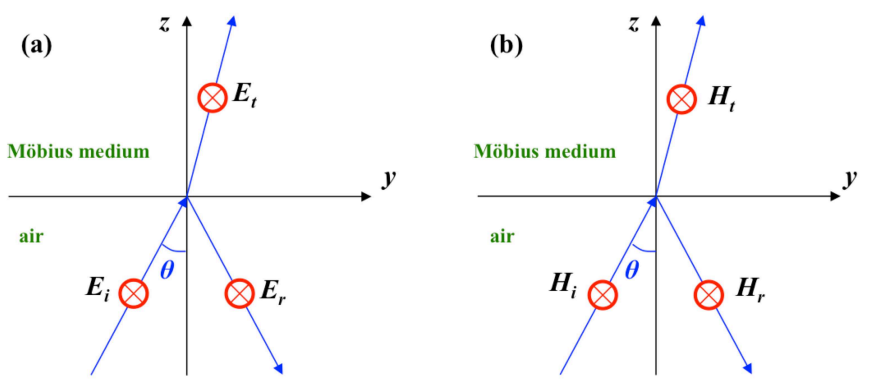

FIG. 3: (color online) Schematic plots of the reflection and the refraction at the medium interface $z=0$. (a) The electric field and (b) the magnetic field of the refracted light is polarized along the $x$-direction, respectively.

wave vector and the $z$ axis. We consider two independent incident configurations respectively:

$$
\vec{E}_{i}=E_{0} \hat{e}_{x} e^{i\left(k_{i y} y+k_{i z} z-\omega t\right)},(\text { E-polarized })
$$

and

$$
\vec{H}_{i}=H_{0} \hat{e}_{x} e^{i\left(k_{i y} y+k_{i z} z-\omega t\right)},(\text { H-polarized })
$$

where $\vec{k}_{i}=k_{i y} \hat{e}_{y}+k_{i z} \hat{e}_{z}$ is the wave vector of incident light. These two configurations are known as E-polarized and $H$-polarized respectively, as the electric and magnetic fields of the refracted light are perpendicular to the refracted wave vector [33], respectively.

\section{A. E-polarized Incident Configuration}

For the specific incident configuration given by Eq. (33), the three criteria for a "left-handed" medium could be checked one by one. It follows from the boundary conditions derived from the Maxwell's equations that [28]

$$
\begin{aligned}
\hat{e}_{z} \times\left(\vec{E}_{i}+\vec{E}_{r}-\vec{E}_{t}\right) & =0 \\
\hat{e}_{z} \times\left(\vec{H}_{i}+\vec{H}_{r}-\vec{H}_{t}\right) & =0 \\
k_{i y}=k_{t y} & =k_{i} \sin \theta,
\end{aligned}
$$

where the subscript $r$ denotes the reflected field back to the air. In accordance with above boundary conditions, the electric fields of the reflected and refracted lights could be written as

$$
\begin{aligned}
\vec{E}_{r} & =r E_{0} \hat{e}_{x} e^{i\left(k_{i y} y-k_{i z} z-\omega t\right)}, \\
\vec{E}_{t} & =t E_{0} \hat{e}_{x} e^{i\left(k_{i y} y+k_{t z} z-\omega t\right)},
\end{aligned}
$$

where we also use $r$ and $t$ to represent the reflection and refraction coefficients respectively, and $1+r=t$. To determine the refracted wave vector, we combine the two Maxwell's equations $\vec{k}_{t} \times \vec{E}_{t}=\omega \mu_{0} \overleftrightarrow{\mu_{r}} \vec{H}_{t}$ and $\vec{k}_{t} \times \vec{H}_{t}=$ $-\omega \varepsilon_{0} \overleftrightarrow{\varepsilon_{r}} \vec{E}_{t}$ : by multiplying both hand sides of the first 
equation with ${\overleftrightarrow{\mu_{r}}}^{-1}$ and then inserting it to the second equation, the following equation could be derived, i.e.

$$
\vec{k}_{t} \times\left[\left(\overleftrightarrow{\mu_{r}}\right)^{-1}\left(\vec{k}_{t} \times \vec{E}_{t}\right)\right]=-\omega^{2} \mu_{0} \varepsilon_{0} \overleftrightarrow{\varepsilon_{r}} \vec{E}_{t}
$$

Since the equation is homogeneous in $E_{t x}, E_{t y}$ and $E_{t z}$, the necessary condition for non-vanishing refracted light is that the determinant of its coefficient matrix is zero. Combined with $\vec{E}_{t}=E_{t x} \hat{e}_{x}$ and $k_{t x}=0$, this necessary condition could be explicitly written as

$$
\frac{\omega^{2}}{c^{2}} \varepsilon_{r}^{(x x)}-\frac{1}{\mu_{1}}\left(k_{i y}^{2} \mu_{r}^{(x x)}+2 \mu_{r}^{(y z)} k_{i y} k_{t z}+\mu_{r}^{(z z)} k_{t z}^{2}\right)=0 .
$$

Inserting Eqs. (26]29) into the above equation yields

$$
\begin{aligned}
k_{t z}= & \left\{k_{i} \sqrt{\mu_{1}\left[\left(1-\eta^{\prime}\right)\left(1-4 \beta^{2} \eta^{\prime}\right)-\sin ^{2} \theta\right]}\right. \\
& \left.-\mu_{r}^{(y z)} k_{i y}\right\} / \mu_{r}^{(z z)} .
\end{aligned}
$$

The time-averaged Poynting vector of refracted light $\vec{S}_{t}=\operatorname{Re}\left\{\vec{E}_{t} \times \vec{H}_{t}^{*}\right\} / 2$ reads

$$
\vec{S}_{t}=S_{t y} \hat{e}_{y}+S_{t z} \hat{e}_{z}
$$

where

$$
\begin{aligned}
& S_{t y}=\frac{E_{0}^{2} t^{2}}{2 \omega \mu_{0} \mu_{1}}\left(k_{t z} \mu_{r}^{(y z)}+k_{i y} \mu_{r}^{(x x)}\right), \\
& S_{t z}=\frac{E_{0}^{2} t^{2}}{2 \omega \mu_{0} \mu_{1}}\left(k_{i y} \mu_{r}^{(y z)}+k_{t z} \mu_{r}^{(z z)}\right) .
\end{aligned}
$$

According to Eqs. (29)40|41), for the E-polarized configuration, the three criteria for "left-handed" medium are specified as $\vec{k}_{t} \cdot \vec{S}_{t}<0$, and

$$
\begin{aligned}
& \left(1-\eta^{\prime}\right)\left(1-4 \beta^{2} \eta^{\prime}\right) \geq \sin ^{2} \theta, \text { if } \mu_{1}>0, \\
& \left(1-\eta^{\prime}\right)\left(1-4 \beta^{2} \eta^{\prime}\right) \leq \sin ^{2} \theta, \text { if } \mu_{1}<0,
\end{aligned}
$$

and

$$
S_{t z}=\frac{E_{0}^{2} t^{2}}{2 \omega \mu_{0} \mu_{1}}\left[-2 \alpha \beta \eta^{\prime} k_{i y}+\left(1-4 \beta^{2} \eta^{\prime}\right) k_{t z}\right]>0 .
$$

Figure 4(a) shows the "phase diagram" in the $\theta-\omega$ plane as determined by the above three criteria. Obviously, there is a frequency window in which the applied electromagnetic fields can be negatively refracted, while in most regions of the $\theta-\omega$ plane the refracted light are "righthanded". Besides these, there is also a considerable region where the light will be totally reflected. The inset of Fig. 4(a) also shows $\mu_{1}$ near the inter-band transition frequency of the individual Möbius molecule. A comparison shows that there is a correspondence between the "lefthanded" phase boundary and the zeros of $\mu_{1}$, which will be illustrated later.

To quantitatively understand the "phase diagram", we rely on the geometry relation between the Poynting vector and the wave vector surface 33]. The wave vector surface is defined by solutions to Eq. (38) in the $\left(k_{t x}, k_{t y}, k_{t z}\right)$ space (or equivalently in the $\left(n_{t x}, n_{t y}, n_{t z}\right)$ space by $\left.\vec{n}_{t} \equiv \vec{k}_{t} / \omega \sqrt{\mu_{0} \varepsilon_{0}}\right)$. This geometry relation then asserts: in the medium with symmetric $\overleftrightarrow{\varepsilon_{r}}$ and $\overleftrightarrow{\mu_{r}}$, the Poynting vector for a given $\vec{k}$ is either parallel or antiparallel to the normal vector of the wave vector surface, as proven in Appendix $\mathrm{E}$.

The shape of the wave vector surface, as shown in Fig. 5. strongly depends on the incident frequency. And those different shapes will in turn give rise to contrasting propagation properties of the refracted fields. Let us first consider an incident field with frequency $\omega \ll \Delta_{0 \uparrow}$. It follows from Eq. (27) that in this case $\eta^{\prime}<0$ and $\left|\eta^{\prime}\right|$ is of the order of unity, while $\alpha \ll 1$ and $\beta \ll 1$. Therefore, after ignoring the second order terms of $\alpha$ and $\beta$ in Eq. (39), the wave vector surface is nearly of circle shape, i.e.,

$$
n_{t y}^{2}+n_{t z}^{2} \approx 1-\eta^{\prime} .
$$

Obviously, a real solution of $k_{t z}$ is admitted for all incident angles, cf. Fig. 5. Since $\mu_{r}^{(y z)} \ll 1$ and meanwhile $\mu_{1}, \mu_{r}^{(x x)}$, and $\mu_{r}^{(z z)}$ are of order one when $\omega \ll \Delta_{0 \uparrow}$, it follows from Eqs. (41,43) that the Poynting vector $\vec{S}_{t}$ is approximately along the direction of $\vec{k}_{t}$. Because $\vec{k}_{t} \cdot \vec{S}_{t}<0$ is violated, the refracted field is "right-handed".

When the frequency of the incident field is increased to just above the lowest inter-band transition frequency $\omega \gtrsim \Delta_{0 \uparrow}$, since $\eta^{\prime} \gg 1, \mu_{1}$ in Eq. (32) could be negative. By linearly combining $n_{t y}$ and $n_{t z}$ to eliminate the cross term in Eq. (39), the wave vector surface is a hyperboloid, i.e.,

$$
\frac{\tilde{n}_{t z}^{2}}{\left(1-\eta^{\prime}\right) \mu_{1}}-\frac{\tilde{n}_{t y}^{2}}{\eta^{\prime}-1}=1,
$$

where $\tilde{n}_{t z}=n_{t z} \sin \phi+n_{t y} \cos \phi, \tilde{n}_{t y}=n_{t z} \cos \phi-n_{t y} \sin \phi$, and the mixing angle $\phi=\tan ^{-1}\left[-4 \alpha \beta /\left(\beta^{2}-\alpha^{2}\right)\right] / 2$. Similar non-closed wave vector surface has been suggested in uniaxial left-handed materials [34]. As $\mu_{1}<0$, Eq. (41) implies that the Poynting vector is opposite to the normal vector of the wave vector surface and thus $\vec{k}_{t} \cdot \vec{S}_{t}<0$, showing that the wave propagating in the Möbius medium is now "left-handed". Furthermore, in order to fulfill the requirement for causality, the refracted wave vector should be on the lower branch, cf. Fig. 5 .

Equation (48) also indicates the frequency region where "left-handed" propagating wave is allowed in the Möbius medium. Since $\alpha$ and $\beta$ are small quantities, the sign of $\mu_{1}$ will change along with the increase of $\eta^{\prime}$. This sign change will make the shape of the wave vector surface deform from a hyperboloid to a spheroid. However, because $1-\eta^{\prime}$ is still negative and $\mu_{1}>0$, Eq. (48) will not have solution for real $n_{t z}$. An imaginary $n_{t z}$ indicates that the wave can not propagate inside the medium and the incident wave is totally reflected. Therefore, the frequency region for the propagating "left-handed" $E$ polarized wave in the Möbius medium is determined by the sign change of $\mu_{1}$, cf. Fig. 4 (a), and the resulting 

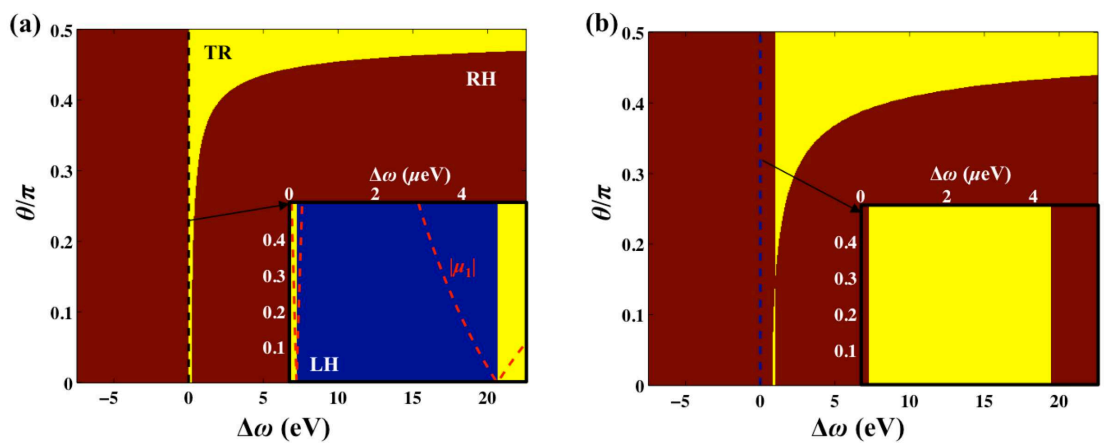

FIG. 4: (color online) "Phase diagram" of left-handedness in the $\theta$ - $\omega$ plane for (a) E-polarized incident field, (b) H-polarized incident field. Red and yellow regions indicate respectively the parameter regions where the refracted wave is "right-handed" $(\mathrm{RH})$ and the incident field is totally reflected (TR) back to the air. The refracted field is "left-handed" (LH) in the blue region. The inserts show a magnified part in the main plot where $\omega$ is close to the lowest inter-band transition. The red dashed curve shows $\left|\mu_{1}\right|$ as a function of $\omega$ near the inter-band transition. Here we use the same parameters as in Fig. 2

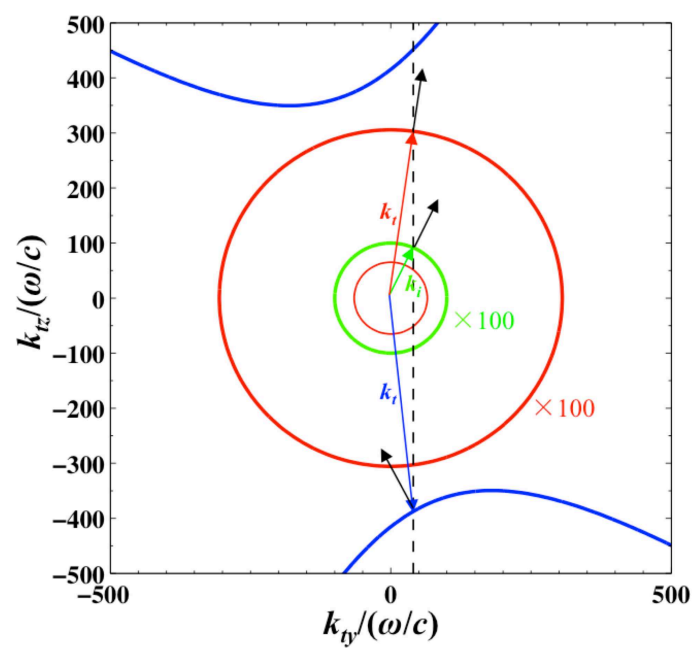

FIG. 5: (color online) Cross sections of the wave vector surfaces of the refracted fields at different incident frequencies: $\omega \ll \Delta_{0 \uparrow}$ (thick red, magnified by 100), $\omega \gtrsim \Delta_{0 \uparrow}$ (thick blue), $\omega \gg \Delta_{0 \uparrow}$ (thin red). The green circle, magnified by 100, depicts the wave vector surface of the incident field ( $E$ polarized). In each case, the refracted wave vector $\vec{k}_{t}$ and the direction of corresponding Poynting vector $\vec{S}_{t}$ (black arrow) are explicitly shown, while the wave vector of the incident field $\vec{k}_{i}=\omega \sqrt{\mu_{0} \omega_{0}}\left(\sin \theta \hat{e}_{y}+\cos \theta \hat{e}_{z}\right)$ is depicted as the green arrow. The black dashed line represents the constraint from the boundary condition. Here we use the same parameters as in Fig. 2 .

bandwidth, i.e., width of the frequency region supporting negative refraction, is $\mathcal{B}=\left|\omega_{1}-\omega_{2}\right|$, where $\omega_{j}$ s are the two solutions to $\mu_{1}\left(\omega_{j}\right)=0$.

If we further tune the frequency $\omega$ to be far bigger than $\Delta_{0 \uparrow}, \eta^{\prime}$ approaches zero, i.e. $\eta^{\prime} \rightarrow 0^{+}$. Equation (47) is again valid in this case, but the radius of the wave vector surface for the refracted field is smaller than that of the incident field. However, $\vec{k}_{t} \cdot \vec{S}_{t}>0$ suggests that the refracted light is also "right-handed" in this case.
Furthermore, the total reflection of the incident light will happened as there is no real solution for $n_{t z}$ at large incident angle.

\section{B. H-polarized Incident Configuration}

The analysis for the $H$-polarized case is similar to that for the E-polarized case. In accordance with Eqs. (35]34), the reflected and refracted magnetic fields are written as, cf. Fig. 3(b)

$$
\begin{aligned}
\vec{H}_{r} & =r H_{0} \hat{e}_{x} e^{i\left(k_{i y} y-k_{i z} z-\omega t\right)}, \\
\vec{H}_{t} & =t H_{0} \hat{e}_{x} e^{i\left(k_{i y} y+k_{t z} z-\omega t\right)} .
\end{aligned}
$$

The equation for determining the refracted wave vector component $k_{t z}$ is similar to Eq. (38), i.e.,

$$
\vec{k}_{t} \times\left[\left(\overleftrightarrow{\varepsilon_{r}}\right)^{-1}\left(\vec{k}_{t} \times \vec{H}_{t}\right)\right]=-\omega^{2} \mu_{0} \varepsilon_{0}{\overleftrightarrow{\mu_{r}}}_{H_{t}}
$$

By solving this equation, we obtain the refracted wave vector as

$$
\begin{aligned}
k_{t z}= & \left\{ \pm k_{i} \sqrt{\varepsilon_{1}\left[\left(1-\eta^{\prime} \alpha^{2}\right)\left(1-4 \eta^{\prime}\right)-\sin ^{2} \theta\right]}\right. \\
& \left.-\varepsilon_{r}^{(y z)} k_{i y}\right\} / \varepsilon_{r}^{(z z)} .
\end{aligned}
$$

The time-averaged Poynting vector for the refracted light is $\vec{S}_{t}=S_{t y} \hat{e}_{y}+S_{t z} \hat{e}_{z}$, with

$$
S_{t y}=\frac{H_{0}^{2} t^{2}}{2 \omega \varepsilon_{0} \varepsilon_{1}}\left(k_{t z} \varepsilon_{r}^{(y z)}+k_{i y} \varepsilon_{r}^{(x x)}\right),
$$

and

$$
S_{t z}=\frac{H_{0}^{2} t^{2}}{2 \omega \varepsilon_{0} \varepsilon_{1}}\left(k_{i y} \varepsilon_{r}^{(y z)}+k_{t z} \varepsilon_{r}^{(z z)}\right) .
$$

The three criteria for "left-handed" medium are then summarized as $\vec{k}_{t} \cdot \vec{S}_{t}<0$, and

$$
\begin{aligned}
& \left(1-\eta^{\prime} \alpha^{2}\right)\left(1-4 \eta^{\prime}\right) \geq \sin ^{2} \theta, \text { if } 5 \eta^{\prime}<1, \\
& \left(1-\eta^{\prime} \alpha^{2}\right)\left(1-4 \eta^{\prime}\right) \leq \sin ^{2} \theta, \text { if } 5 \eta^{\prime}>1
\end{aligned}
$$


and

$$
\left(1-5 \eta^{\prime}\right)\left[-2 \eta^{\prime} k_{i y}+\left(1-4 \eta^{\prime}\right) k_{t z}\right]>0 .
$$

The "phase diagram" in the $\theta-\omega$ plane for the $H$ polarized case is deduced according to above three criteria, as shown in Fig. 4(b). Similarly to the E-polarized case, there are regions in the $\theta-\omega$ plane where the refracted propagating field is "right-handed" and regions where the incident field is totally reflected. However, in contrast to the E-polarized case, there are no frequency regions in the $H$-polarized case such that the propagating refracted field could be "left-handed".

\section{Discussions}

In the previous subsections, we have discussed the negative refraction regardless of loss. Generally speaking, there will be loss in the medium due to couplings of the molecules to the bath. The condition for negative refraction in the presence of loss is slightly different from that without loss [37, 38]. Because of the loss effect, the imaginary part $\eta^{\prime \prime}$ should also be considered in the relative permittivity and permeability. This results in the replacement of $\eta^{\prime}$ by $\eta=\eta^{\prime}+i \eta^{\prime \prime}$ in Eqs. (26]29). Secondly, the refracted wave vector as determined from the Maxwell's equations is now generally complex, and the phase velocity is along the real part of the refracted wave vector [37, 38]. Through considering the normal incidence of an E-polarized field onto the medium interface, we can prove that the frequency region of negative refraction is not qualitatively changed when the loss is taken into consideration.

On the other hand, as depicted in Eq. (27), the negative refraction will apparently disappear if the decay rate of excited stats is sufficiently large. A limitation on the lifetime of the excited state for achieving negative refraction could be deduced from the bandwidth $\mathcal{B}=\left|\omega_{1}-\omega_{2}\right|$ according to zeros of $\mu_{1}(\omega)$, i.e.

$$
\mathcal{B}=\operatorname{Re} \sqrt{\left[\frac{e^{2}\left(\alpha^{2}+4 \beta^{2}\right) W^{2}}{8 \varepsilon_{0} v_{0} \hbar}\right]^{2}-4 \gamma^{2}} .
$$

This indicates a restriction on the excited state lifetime

$$
\tau_{c}=\frac{16 \varepsilon_{0} v_{0} \hbar}{e^{2}\left(\alpha^{2}+4 \beta^{2}\right) W^{2}},
$$

above which the negative refraction from the Möbius medium is expectable. Numerical verification has been performed and shows that the bandwidth is not qualitatively changed with the inclusion of the loss effect. In experiments, it is possible to synthesize a Möbius ring of carbon atoms with $N=12$ and radius $0.29 \mathrm{~nm}$ [21, 22]. And it was theoretically predicted that Möbius molecules with more than 60 atoms are as stable as Hückel molecules [41]. By taking the value $3.6 \mathrm{eV}$ for $V$ and $\xi$ [31], one finds that an excited state lifetime of
$0.51 \mathrm{~ns}$ is enough for observing negative refraction. Since the excited-state lifetime of Möbius systems can reach as long as 350ns [39, 40], it is reasonable to expect a medium with Möbius molecules as a potential material to show the negative refraction.

Although in our calculation only one electron is considered, our result is consistent with the more realistic case when all $\pi$ electrons from all atoms in the Möbius molecule are taken into account. In that case, when the spin degree of freedom is considered, two electrons with different spin states can stay in the same energy eigenstate $|k, \sigma\rangle$. For the ground state of the total system including all electrons, all the states of the lower energy band will be filled. Theoretically, there could be negative refraction around $4 N$ possible transition frequencies if the excited-state lifetime is sufficiently long, as implied by Eqs. (16/21/58). The inter-band transition in our calculation is just the special case of $4 N$ possible transitions. In this sense, our simplified calculation clearly illustrates the key factors for demonstrating negative refraction in Möbius molecules.

Furthermore, the Hamiltonian describing a molecule interacting with electromagnetic field is approximated as dipole interaction in our calculation, cf. Eqs. (12/20). Generally speaking, there are multi-pole contributions to Coulomb interaction between molecule and electromagnetic field. As long as the molecule is small, the dipole approximation is valid and has been frequently used in the investigation of metamaterials [14, 28]. On the other hand, for the sake of simplicity, the inter-molecular interaction has been neglected in our calculation. As shown in the Appendix E $\mathrm{E}$ based on the Lorentz local field theory, both the central frequency and bandwidth of negative refraction have not been substantially modified when the interaction between molecules is taken into account. As a result, by modeling Möbius molecules as non-interacting particles, the key factors influencing negative refraction can be captured.

The Möbius molecule discussed in this paper is termed an equilateral Möbius strip as the twist density is the same everywhere. In Ref. [25], it was reported that two conformations of tetrahydroxymethylethylene Möbius molecule with chemical formula $\mathrm{C}_{42} \mathrm{H}_{72} \mathrm{O}_{18}$ have been synthesized. Although the equilateral Möbius molecule has not yet been synthesized, it was predicted that it could be achievable 25]. On the other hand, we notice that the Hückel model with empirical parameters has been successfully applied to describing experimental data of more than 60 organic molecules with maximum deviation no more than $15 \%$ [31, 32, 43]. Therefore, it is reasonable to expect that the theoretical predictions for Möbius molecules could also be observed in the future experiments. 


\section{CONCLUSION}

We have explored the Möbius molecular ring as a potential candidate for negative refraction. The previous investigations with the functional atoms or molecules rely on the conceptual analogy of the split-ring resonator for magnetic response [15], while for Möbius ring this is induced by its non-trivial boundary condition. Our results demonstrate that engineering on the topology is beneficial in realizing the high frequency magnetic response at the molecular level. This finding opens up an alternative approach to design molecular negative index materials, which is promising in achieving $3 \mathrm{D}$ bulk negative refraction at the visible wavelength.

We further remark that our proposal is complementary to the previous experimental investigation [10], where the classical metamaterial was fabricated with Möbius topology in a "top-down" fashion. In order to induce the magnetic response, their element is still based on the configuration of the split-ring resonator. Moreover, due to quantum effect, our architecture is two order smaller in size than theirs.

This work was supported by the National Natural Science Foundation of China (Grant No. 11121403 and No. 11505007), the National 973-program (Grant No. 2012CB922104 and No. 2014CB921403), and the Youth Scholars Program of Beijing Normal University (Grant No. 2014NT28), and the Open Research Fund Program of the State Key Laboratory of LowDimensional Quantum Physics, Tsinghua University Grant No. KF201502.

\section{Appendix A: Absence of Magnetic Dipole Transition in a Perfect Ring}

Consider a molecular ring formed by $N$ identical atoms with the nearest neighbour hopping strength $\xi$ and site energy $\epsilon$. The single electron Hamiltonian is written as

$$
H=\sum_{j=0}^{N-1}\left[\epsilon a_{j}^{\dagger} a_{j}-\left(\xi a_{j}^{\dagger} a_{j+1}+\text { h.c. }\right)\right]
$$

where periodical boundary condition $a_{0}=a_{N}$ is assumed. The operator $a_{j}^{\dagger}$ creates an excitation at the $j$ th atom, which is located at $\vec{R}_{j}=R \cos \varphi_{j} \hat{e}_{x}+R \sin \varphi_{j} \hat{e}_{y}$ and $\varphi_{j}$ is defined in Eq. (15), $R$ denotes the radius of the molecular ring.

Based on the bond current formalism in Ref. [27], the magnetic dipole operator $\vec{m}$ reads

$$
m_{x}=m_{y}=0, m_{z}=\frac{i}{2} e \xi R^{2} \sin \delta \sum_{j} a_{j}^{\dagger} a_{j+1}+\text { h.c. }
$$

Equations A1A2 indicate that a perfect ring does not couple to the magnetic field [17]. Because $[\vec{m}, H]=0$ and the interaction Hamiltonian is proportional to $\vec{m}$, the interaction does not mix different eigenstates of $H$. Consequently, the negative refraction is absent in this situation.

Furthermore, we can show that for a common double ring, i.e., a chemical annulene with the periodical boundary condition $a_{0}=a_{N}$ and $b_{0}=b_{N}$, the magnetic-dipole transition is not at the same frequency as the electricdipole transition. As a result, there will not be the negative refraction either.

\section{Appendix B: Diagonalization of Möbius Hamiltonian}

Before evaluating matrix elements for dipole operators, we solve explicitly the energy spectrum and the molecular eigenstates. Consider a Möbius molecular ring with $2 N$ sites, which is described by the Hückel Hamiltonian $H$ as illustrated in Eq. (11). The Hamiltonian $H$ is in diagonal form when expressed in terms of $\mathbf{C}_{k}$ and $\mathbf{C}_{k}^{\dagger}$, i.e.,

$$
H=\sum_{k} \mathbf{C}_{k}^{\dagger} \mathbf{E}_{k} \mathbf{C}_{k}
$$

with

$$
\begin{aligned}
& \mathbf{C}_{k}=\left[\begin{array}{l}
d_{k \uparrow} \\
d_{k \downarrow}
\end{array}\right]=\frac{1}{\sqrt{N}} \sum_{j=0}^{N-1} e^{i k j} \mathbf{B}_{j}, \\
& \mathbf{E}_{k}=\left[\begin{array}{cc}
V-2 \xi \cos \left(k-\frac{\delta}{2}\right) & 0 \\
0 & -V-2 \xi \cos k
\end{array}\right] .
\end{aligned}
$$

Therefore, the single-electron molecular eigenstates $|k, \sigma\rangle$ are

$$
|k, \uparrow\rangle=d_{k \uparrow}^{\dagger}|0\rangle,|k, \downarrow\rangle=d_{k \downarrow}^{\dagger}|0\rangle .
$$

Here, $|0\rangle$ denotes the vacuum state and $k=l \delta$ with $l=$ $0,1, \ldots, N-1$ and $\delta=2 \pi / N$.

It is useful to express $|k, \sigma\rangle$ in terms of the localized atomic-orbitals for later use. To achieve this one notice that, according to Ref. [19], $\mathbf{B}_{j}$ is related to $\mathbf{A}_{j}$ by a local unitary transformation, i.e.

$$
\mathbf{B}_{j}=\left[\begin{array}{c}
c_{j+} \\
c_{j-}
\end{array}\right]=\mathbf{U}_{j} \mathbf{A}_{j}
$$

with

$$
\mathbf{U}_{j}=\frac{1}{\sqrt{2}}\left[\begin{array}{cc}
e^{-i \varphi_{j} / 2} & -e^{-i \varphi_{j} / 2} \\
1 & 1
\end{array}\right],
$$

and $j=0,1, \ldots, N-1$. It follows from Eqs. (B/B5) that the eigenstates $|k, \sigma\rangle$ can be written in terms of $\mathbf{A}_{j}=$ $\left[\begin{array}{ll}a_{j} & b_{j}\end{array}\right]^{\mathrm{T}}$ as

$$
\begin{aligned}
{\left[\begin{array}{l}
|k, \uparrow\rangle \\
|k, \downarrow\rangle
\end{array}\right] } & =\sum_{j=0}^{N-1} \frac{e^{-i k j}}{\sqrt{N}}\left[\begin{array}{c}
c_{j \uparrow}^{\dagger} \\
c_{j \downarrow}^{\dagger}
\end{array}\right]|0\rangle \\
& =\sum_{j=0}^{N-1} \frac{e^{-i k j}}{\sqrt{2 N}}\left[\begin{array}{cc}
e^{i \varphi_{j} / 2} & -e^{i \varphi_{j} / 2} \\
1 & 1
\end{array}\right]\left[\begin{array}{c}
a_{j}^{\dagger} \\
b_{j}^{\dagger}
\end{array}\right]|0\rangle .
\end{aligned}
$$


Furthermore, by noticing that the atomic orbitals $\left|\phi_{j \pm}\right\rangle$ are created by acting $a_{j}^{\dagger}$ or $b_{j}^{\dagger}$ on the vacuum state, the molecular eigenstates are rewritten as

$$
\begin{aligned}
& |k, \uparrow\rangle=\frac{1}{\sqrt{2 N}} \sum_{j=0}^{N-1} e^{-i\left(k-\frac{\delta}{2}\right) j}\left(\left|\phi_{j+}\right\rangle-\left|\phi_{j-}\right\rangle\right), \\
& |k, \downarrow\rangle=\frac{1}{\sqrt{2 N}} \sum_{j=0}^{N-1} e^{-i k j}\left(\left|\phi_{j+}\right\rangle+\left|\phi_{j-}\right\rangle\right) .
\end{aligned}
$$

Especially, if the resonance integrals $V$ and $\xi$ are positive, the molecular ground state is

$$
|g\rangle=|0, \downarrow\rangle=\frac{1}{\sqrt{2 N}} \sum_{j=0}^{N-1}\left(\left|\phi_{j+}\right\rangle+\left|\phi_{j-}\right\rangle\right) .
$$

\section{Appendix C: Matrix Elements of Electric and Magnetic Dipoles}

The matrix elements of electric dipole operator are

$$
\begin{aligned}
\left\langle 0\left|\mathbf{C}_{k} \vec{d} \mathbf{C}_{k}^{\dagger}\right| 0\right\rangle= & -e \frac{W}{4}\left[\left(\hat{e}_{y}+2 \hat{e}_{z}\right) \sigma_{x}-\hat{e}_{x} \sigma_{y}\right] \\
\left\langle 0\left|\mathbf{C}_{k} \vec{d} \mathbf{C}_{k \pm \delta}^{\dagger}\right| 0\right\rangle= & -e \frac{1}{4}\left[\left(\hat{e}_{x} \mp i \hat{e}_{y}\right)\left(2 R+W \sigma_{y}\right)\right. \\
& \left.+2 W \hat{e}_{z} \sigma_{\mp}\right] \\
\left\langle 0\left|\mathbf{C}_{k} \vec{d} \mathbf{C}_{k \pm 2 \delta}^{\dagger}\right| 0\right\rangle= & -e \frac{W}{4}\left(\mp i \hat{e}_{x}-\hat{e}_{y}\right) \sigma_{\mp}
\end{aligned}
$$

where $\vec{d}=-e \vec{r}$ is the electric dipole operator with $-e$ being the electric charge and $\vec{r}$ being the position vector of electron, $\sigma_{ \pm}=\left(\sigma_{x} \pm i \sigma_{y}\right) / 2$ with $\sigma_{\alpha}(\alpha=x, y, z)$ being Pauli operators in the pseudo spin space spanned by $|k, \sigma\rangle$ and $\left|k^{\prime}, \sigma\right\rangle$, e.g., for Eq. (C1) $\sigma_{z}$ is defined as

$$
\sigma_{z}=|k, \uparrow\rangle\langle k, \uparrow|-| k, \downarrow\rangle\langle k, \downarrow|,
$$

$\hat{e}_{\alpha}(\alpha=x, y, z)$ is the unit vector in $\alpha$ direction, $R$ and $4 W$ are respectively the radius and width of the Möbius molecule.

The matrix elements of magnetic dipole operator $\vec{m}=$ $-i e \vec{r} \times[H, \vec{r}] / 2 \hbar$ are summarized as follows:

For $|k, \sigma\rangle \rightleftharpoons\left|k, \sigma^{\prime}\right\rangle$ transitions

$$
\begin{gathered}
-\left\langle 0\left|\mathbf{C}_{k} \vec{m} \mathbf{C}_{k}^{\dagger}\right| 0\right\rangle \\
=\frac{e}{2 \hbar}\left[\begin{array}{c}
-\frac{1}{8} \xi\left\{2 W^{2}[\cos (k-\delta)-\cos k] \hat{e}_{y}+\left[W^{2}(\cos k\right.\right. \\
-\cos (k-2 \delta)-\cos (k-\delta)+\cos (k+\delta)) \\
\left.\left.+4 R^{2}\left(\cos \left(k+\frac{\delta}{2}\right)-\cos \left(k-\frac{3}{2} \delta\right)\right)\right] \hat{e}_{z}\right\} \\
\frac{1}{4} R W\left\{-\left[V+\xi\left(\cos (k-\delta)-\cos \left(k+\frac{\delta}{2}\right)\right]\left(\hat{e}_{x}+i \hat{e}_{y}\right)\right.\right. \\
\left.-2 i \xi \cos \frac{\delta}{4}\left[\cos \left(k+\frac{3}{4} \delta\right)-\cos \left(k-\frac{5}{4} \delta\right)\right] \hat{e}_{z}\right\}
\end{array}\right.
\end{gathered}
$$$$
\left.\begin{array}{c}
\frac{1}{4} R W\left\{-\left[V+\xi\left(\cos (k-\delta)-\cos \left(k+\frac{\delta}{2}\right)\right)\right]\left(\hat{e}_{x}-i \hat{e}_{y}\right)\right. \\
\left.-2 i \xi \cos \frac{\delta}{4}\left[\cos \left(k-\frac{5}{4} \delta\right)-\cos \left(k+\frac{3}{4} \delta\right)\right] \hat{e}_{z}\right\} \\
-\frac{1}{2} \xi \sin k\left[W^{2} \sin \frac{\delta}{2} \hat{e}_{y}-\left(2 R^{2}+W^{2} \cos \frac{\delta}{2}\right) \sin \delta \hat{e}_{z}\right]
\end{array}\right],
$$

for $|k, \sigma\rangle \rightleftharpoons\left|k \pm \delta, \sigma^{\prime}\right\rangle$ transitions

$$
\begin{aligned}
& -\left\langle 0\left|\mathbf{C}_{k} \vec{m} \mathbf{C}_{k+\delta}^{\dagger}\right| 0\right\rangle \\
= & \frac{e}{2 \hbar}\left[\begin{array}{cc}
\frac{1}{8} W^{2} \xi[\cos (k-\delta)-\cos (k+\delta)]\left(i \hat{e}_{x}+\hat{e}_{y}-\hat{e}_{z}\right) & -\frac{1}{4} R W\left\{\left[V+\xi\left(\cos k-\cos \left(k+\frac{\delta}{2}\right)\right)\right]\left(\hat{e}_{x}-i \hat{e}_{y}\right)\right. \\
\frac{1}{4} R W\left\{\left[V+\xi\left(\cos (k+\delta)-\cos \left(k-\frac{\delta}{2}\right)\right)\right]\left(\hat{e}_{x}-i \hat{e}_{y}\right)\right. & \left.-2 i \xi \cos \frac{\delta}{4}\left[\cos \left(k-\frac{5}{4} \delta\right)-\cos \left(k+\frac{3}{4} \delta\right)\right] \hat{e}_{z}\right\} \\
-i \xi\left[\cos (k-\delta)-\cos (k+\delta)-\cos \left(k+\frac{3}{2} \delta\right)\right. & \frac{1}{8} W^{2} \xi\left[\cos \left(k-\frac{\delta}{2}\right)-\cos \left(k+\frac{3}{2} \delta\right)\right]\left(i \hat{e}_{x}+\hat{e}_{y}-\hat{e}_{z}\right) \\
\left.\left.+\cos \left(k-\frac{\delta}{2}\right)\right] \hat{e}_{z}\right\} &
\end{array}\right],
\end{aligned}
$$


and

$$
\begin{aligned}
& -\left\langle 0\left|\mathbf{C}_{k} \vec{m} \mathbf{C}_{k-\delta}^{\dagger}\right| 0\right\rangle \\
= & \frac{e}{2 \hbar}\left[\begin{array}{cc}
-\frac{1}{8} W^{2} \xi[\cos (k-2 \delta)-\cos k]\left(i \hat{e}_{x}-\hat{e}_{y}+\hat{e}_{z}\right) & \frac{1}{4} R W\left\{\left[V+\xi\left(\cos k-\cos \left(k-\frac{3}{2} \delta\right)\right)\right]\left(\hat{e}_{x}+i \hat{e}_{y}\right)\right. \\
-\frac{1}{4} R W\left\{V+\xi\left[\cos (k-\delta)-\cos \left(k-\frac{\delta}{2}\right)\right]\right\}\left(\hat{e}_{x}+i \hat{e}_{y}\right) & \frac{1}{8} W^{2} \xi\left[\cos \left(k-\frac{3}{2} \delta\right)-\cos \left(k+\frac{\delta}{2}\right)\right]\left(-i \hat{e}_{x}+\hat{e}_{y}-\hat{e}_{z}\right)
\end{array}\right] .
\end{aligned}
$$

for $|k, \sigma\rangle \rightleftharpoons\left|k \pm 2 \delta, \sigma^{\prime}\right\rangle$ transitions

$$
\begin{aligned}
& -\left\langle 0\left|\mathbf{C}_{k} \vec{m} \mathbf{C}_{k+2 \delta}^{\dagger}\right| 0\right\rangle \\
= & \frac{e}{2 \hbar}\left[\begin{array}{cc}
\frac{i}{8} W^{2} \xi[\cos k-\cos (k+\delta)]\left(\hat{e}_{x}-i \hat{e}_{y}\right) & 0 \\
\frac{1}{4} R W\left\{\begin{array}{l}
\text { V } \\
V
\end{array}\left[\cos (k+\delta)-\cos \left(k+\frac{\delta}{2}\right)\right]\right\}\left(\hat{e}_{x}-i \hat{e}_{y}\right) & \frac{i}{8} W^{2} \xi\left[\cos \left(k+\frac{\delta}{2}\right)-\cos \left(k+\frac{3}{2} \delta\right)\right]\left(\hat{e}_{x}-i \hat{e}_{y}\right)
\end{array}\right],
\end{aligned}
$$

and

$$
\begin{aligned}
& -\left\langle 0\left|\mathbf{C}_{k} \vec{m} \mathbf{C}_{k-2 \delta}^{\dagger}\right| 0\right\rangle \\
= & \frac{e}{2 \hbar}\left[\begin{array}{cc}
-\frac{i}{8} W^{2} \xi[\cos (k-2 \delta)-\cos (k-\delta)]\left(\hat{e}_{x}+i \hat{e}_{y}\right) & \frac{1}{4} R W\left\{V+\xi\left[\cos (k-\delta)-\cos \left(k-\frac{3}{2} \delta\right)\right]\right\}\left(\hat{e}_{x}+i \hat{e}_{y}\right) \\
0 & -\frac{i}{8} W^{2} \xi\left[\cos \left(k-\frac{3}{2} \delta\right)-\cos \left(k-\frac{\delta}{2}\right)\right]\left(\hat{e}_{x}+i \hat{e}_{y}\right)
\end{array}\right],
\end{aligned}
$$

where $V$ and $\xi$ are respectively the inter-ring and intraring resonance integrals, $k$ is the momentum of the eigenstate, $\delta=2 \pi / N$.

\section{Appendix D: Polarization and Magnetization}

In order to calculate the polarization of medium, we first consider the electric dipole $\langle\vec{d}\rangle$ of a single molecule. According to the linear response theory [42]

$$
\langle\vec{d}\rangle=\int \frac{d \omega_{1}}{2 \pi} S^{(1)}\left(\omega_{1}\right) \vec{E}\left(\omega_{1}\right) e^{-i \omega_{1} t},
$$

where $\vec{E}\left(\omega_{1}\right)$ is the Fourier transform of the electric field

$$
\vec{E}\left(\omega_{1}\right)=\int_{-\infty}^{\infty} d t \vec{E}(t) e^{i \omega_{1} t}
$$

and the linear response function in the frequency domain is

$$
S^{(1)}\left(\omega_{1}\right)=-J\left(\omega_{1}\right)-J^{*}\left(-\omega_{1}\right) .
$$

Here, $J\left(\omega_{1}\right)$ is the dipole-dipole correlation function 42]

$$
J\left(\omega_{1}\right)=-i \int_{0}^{\infty} d t \operatorname{Tr}\left[\vec{d}(t) \vec{d} \rho_{0}\right] e^{i \omega_{1} t}
$$

with $\rho_{0}=|g\rangle\langle g|$ and

$$
\vec{d}(t)=e^{i H t / \hbar} \overrightarrow{d e^{-i H t / \hbar}} .
$$

For a monochromatic continuous driving with frequency $\omega$ and time-independent envelope $\vec{E}_{0}$, the electric field is

$$
\vec{E}(t)=\vec{E}_{0} \cos \omega t
$$

and $\langle\vec{d}\rangle$ is rewritten as

$$
\begin{aligned}
\langle\vec{d}\rangle= & \int \frac{d \omega_{1}}{2 \pi} S^{(1)}\left(\omega_{1}\right) \pi \vec{E}_{0}\left[\delta\left(\omega_{1}-\omega\right)+\delta\left(\omega_{1}+\omega\right)\right] e^{-i \omega_{1} t} \\
= & \frac{1}{2}\left[S^{(1)}(\omega) e^{-i \omega t}+S^{(1)}(-\omega) e^{i \omega t}\right] \vec{E}_{0} \\
= & -\frac{1}{2}\left\{\left[J(\omega)+J^{*}(-\omega)\right] e^{-i \omega t}+\left[J(-\omega)+J^{*}(\omega)\right] e^{i \omega t}\right\} \\
& \times \vec{E}_{0} \quad \text { (D7) }
\end{aligned}
$$

According to Eq. (D4), we have

$$
\begin{aligned}
J(\omega) & =-i \int_{0}^{\infty} d t\langle g|\vec{d}(t) \vec{d}| g\rangle \\
& =-i \sum_{k, \sigma}^{\prime} \int_{0}^{\infty} d t e^{i\left(\omega-\Delta_{k \sigma}+i \gamma\right) t} \vec{d}_{g, k \sigma} \vec{d}_{k \sigma, g} \\
& =\sum_{k \sigma}{ }^{\prime} \frac{\vec{d}_{g, k \sigma} \vec{d}_{k \sigma, g}}{\omega-\Delta_{k \sigma}+i \gamma}
\end{aligned}
$$

where $\vec{d}_{k \sigma, g}=\langle k, \sigma|\vec{d}| g\rangle$ is matrix element of the electric dipole operator and the decay rate $\gamma$ (related to the lifetime of the excited states $\tau$ by $\gamma=1 / \tau$ ) is introduced to phenomenologically describe the quantum dynamics due to coupling to the bath. The transition frequency from the ground state to excited state $|k, \sigma\rangle$ is denoted as $\Delta_{k \sigma}$. The electric dipole is then written as 


$$
\begin{aligned}
\langle\vec{d}\rangle & =-\sum_{k, \sigma}, \frac{\vec{d}_{g, k \sigma} \vec{d}_{k \sigma, g} \cdot \vec{E}_{0}}{2 \hbar}\left[\left(\frac{1}{\omega-\Delta_{k \sigma}+i \gamma}-\frac{1}{\omega+\Delta_{k \sigma}+i \gamma}\right) e^{-i \omega t}+\left(\frac{1}{\omega-\Delta_{k \sigma}-i \gamma}-\frac{1}{\omega+\Delta_{k \sigma}-i \gamma}\right) e^{i \omega t}\right] \\
& \approx-\sum_{k, \sigma}{ }^{\prime} \frac{\vec{d}_{g, k \sigma} \vec{d}_{k \sigma, g} \cdot \vec{E}_{0}}{2 \hbar}\left(\frac{1}{\omega-\Delta_{k \sigma}+i \gamma} e^{-i \omega t}+\frac{1}{\omega-\Delta_{k \sigma}-i \gamma} e^{i \omega t}\right),
\end{aligned}
$$

where we have invoked the rotating wave approximation and ignored the terms with $\omega+\Delta_{k \sigma}$ in the denominator [42]. Re-expressing the above equation in terms of $\vec{E}(t)$ yields

$$
\begin{aligned}
\langle\vec{d}\rangle= & -\operatorname{Re} \sum_{k, \sigma}, \frac{\vec{d}_{g, k \sigma} \vec{d}_{k \sigma, g} \cdot \vec{E}(t)}{\hbar\left(\omega-\Delta_{k \sigma}+i \gamma\right)} \\
& +\operatorname{Im} \sum_{k, \sigma}, \frac{\vec{d}_{g, k \sigma} \vec{d}_{k \sigma, g} \cdot \vec{E}\left(t-\frac{\pi}{2 \omega}\right)}{\hbar\left(\omega-\Delta_{k \sigma}+i \gamma\right)} .
\end{aligned}
$$

Since the second term on the r.h.s. of Eq. (D10) is related to absorption and loss, we only keep the real part, i.e.,

$$
\langle\vec{d}\rangle=-\operatorname{Re} \sum_{k, \sigma}{ }^{\prime} \frac{\vec{d}_{g, k \sigma} \vec{d}_{k \sigma, g} \cdot \vec{E}(t)}{\hbar\left(\omega-\Delta_{k \sigma}+i \gamma\right)} .
$$

For a medium of non-interacting molecules, because all molecules contribute equally to the polarization, the polarization is obtained from Eq. (D11) by multiplying it with the molecular number density $1 / v_{0}$, i.e.

$$
\vec{P}(t)=-\frac{1}{\hbar v_{0}} \operatorname{Re} \sum_{k, \sigma}, \frac{\vec{d}_{g, k \sigma} \vec{d}_{k \sigma, g} \cdot \vec{E}(t)}{\omega-\Delta_{k \sigma}+i \gamma} .
$$

For a double-ring Möbius molecule with $N$ carbon atoms in each ring, the radius is $R \simeq N R_{c} / \pi$ and the width $4 W=4 R_{c}$, where $R_{c}=0.077 \mathrm{~nm}[32$ ] is the radius of a single carbon atom. As a result, the volume occupied by a single Möbius molecule is

$$
v_{0} \simeq 2 \pi(R+W)^{2} W .
$$

The magnetization is derived in analogy with Eq. (D12) by noticing the similarity between $H_{B}^{\prime}$ and $H_{E}^{\prime}$, i.e.,

$$
\begin{aligned}
\langle\vec{m}\rangle & =-\operatorname{Re} \sum_{k, \sigma}, \frac{\vec{m}_{g, k \sigma} \vec{m}_{k \sigma, g} \cdot \vec{B}(t)}{\hbar\left(\omega-\Delta_{k \sigma}+i \gamma\right)} \\
\vec{M}(t) & =-\frac{1}{\hbar v_{0}} \operatorname{Re} \sum_{k, \sigma} \frac{\vec{m}_{g, k \sigma} \vec{m}_{k \sigma, g} \cdot \vec{B}(t)}{\omega-\Delta_{k \sigma}+i \gamma} \\
& =-\frac{\mu_{0}}{\hbar v_{0}} \operatorname{Re} \sum_{k, \sigma}, \frac{\vec{m}_{g, k \sigma} \vec{m}_{k \sigma, g} \cdot \vec{H}(t)}{\omega-\Delta_{k \sigma}+i \gamma} .
\end{aligned}
$$

Here, $\vec{m}_{k \sigma, g}=\langle k, \sigma|\vec{m}| g\rangle$ is matrix element of the magnetic dipole operator.

\section{Appendix E: Local Field Correction}

Influenced by polarization of nearby molecules, the actual field that is exerted on an individual molecule could be different from the applied external field. Consequently, the total field at the molecule is modified as

$$
\vec{E}_{\mathrm{tot}}=\vec{E}+\vec{E}_{i}
$$

where $\vec{E}$ and $\vec{E}_{i}$ denote respectively the external and internal fields, and the latter is due to the effect caused by the surrounding molecules. Although the internal field can be obtained by mean-field approximation as

$$
\vec{E}_{\text {mean }}=-\frac{1}{V} \int_{V} d \vec{r} \nabla \Phi(\vec{r}),
$$

where $\Phi(\vec{r})$ is the scalar potential due to charge distribution inside a volume $V$, the approximation fails to take the effect of arrangement of the closest molecules into account. The volume is properly chosen to include a macroscopic number of molecules but still such small enough that the dipole moments for each molecules inside are approximately the same. To account for the arrangement of nearby molecules, the internal field is evaluated by the microscopic contribution $\vec{E}_{\text {near }}$ from all charges of the nearby molecules in $V$ subtracted by the mean-field part. This yields [28]

$$
\vec{E}_{i}=\vec{E}_{\text {near }}-\vec{E}_{\text {mean }} .
$$

However, for molecules arranged with higher symmetry, e.g., cubic lattice, or totally disordered, the contribution from $\vec{E}_{\text {near }}$ can be neglected [28]. Furthermore, by expanding the scalar potential up to the dipole correction and assuming no net charge in the volume $V$, the meanfield part is evaluated as [28]

$$
\vec{E}_{\text {mean }}=-\frac{1}{3 \varepsilon_{0}} \sum_{l} \frac{\vec{p}_{l}}{V},
$$

where $\vec{p}_{l}$ denotes the induced dipole moment of $l$ th molecule inside the volume $V$.

For a sufficiently weak field, the induced dipole moment is proportional to the exerted field, i.e.

$$
\vec{p}_{l}=\varepsilon_{0} \gamma_{\mathrm{mol}} \vec{E}_{\mathrm{tot}},
$$


where the molecular polarizability $\gamma_{\mathrm{mol}}$ is deduced according to Eq. (D11) as

$$
\gamma_{\mathrm{mol}}^{(i j)}=-\frac{1}{\hbar \varepsilon_{0}} \sum_{k, \sigma} \frac{d_{g, k \sigma}^{(i)} d_{k \sigma, g}^{(j)}\left(\omega-\Delta_{k \sigma}\right)}{\left(\omega-\Delta_{k \sigma}\right)^{2}+\gamma^{2}} .
$$

As the volume $V$ is chosen such that its enclosed $\vec{p}_{l}$ s are approximately the same, the polarization within the volume is related to the dipole moment of a representative molecule $\vec{p}_{1}$, multiplied with the molecule density $1 / v_{0}$

$$
\vec{P}=\sum_{l} \frac{\vec{p}_{l}}{V}=\frac{1}{v_{0}} \vec{p}_{1}
$$

On the other hand, the polarization $\vec{P}$ is related to the external field through the electric susceptibility $\vec{P}=\varepsilon_{0} \chi_{e} \vec{E}$. By combining Eqs. (E5 E7), we have

$$
\varepsilon_{0} \chi_{e} \vec{E}=\frac{\varepsilon_{0}}{v_{0}} \gamma_{\mathrm{mol}}\left(\vec{E}+\frac{1}{3 \varepsilon_{0}} \varepsilon_{0} \chi_{e} \vec{E}\right),
$$

from which the electric susceptibility could be obtained. Furthermore, by $\overleftrightarrow{\varepsilon_{r}}=1+\chi_{e}$, the relative permittivity with the local field correction is given by

$$
\begin{aligned}
\overleftrightarrow{\varepsilon_{r}} & =1+\left(1-\frac{1}{3 v_{0}} \gamma_{\mathrm{mol}}\right)^{-1} \frac{1}{v_{0}} \gamma_{\mathrm{mol}} \\
& =\left[\begin{array}{ccc}
\frac{3-2 \eta^{\prime}}{3+\eta^{\prime}} & 0 & 0 \\
0 & \frac{3+2 \eta^{\prime}}{3+5 \eta^{\prime}} & -\frac{6 \eta^{\prime}}{3+5 \eta^{\prime}} \\
0 & -\frac{6 \eta^{\prime}}{3+5 \eta^{\prime}} & \frac{3-7 \eta^{\prime}}{3+5 \eta^{\prime}}
\end{array}\right]
\end{aligned}
$$

where in the second line an approximation similar to the one for Eq. (26) has been used. It follows from Eq. (E9) that the corresponding eigenvalues of $\overleftrightarrow{\varepsilon_{r}}$ are given by $\left(3-2 \eta^{\prime}\right) /\left(3+\eta^{\prime}\right),\left(3-10 \eta^{\prime}\right) /\left(3+5 \eta^{\prime}\right)$ and 1 respectively.

Compared with the results without the local field correction, the bandwidth does not change qualitatively. From relative permittivity, the bandwidth near the lowest inter-band transition frequency $\Delta_{0, \uparrow}$ without local field correction is limited by the separation between two zeros of $1-5 \eta^{\prime}(\omega)=0$, while by including the local field correction, it is limited by two zeros of $1-10 \eta^{\prime}(\omega) / 3=0$. Hence the bandwidth in those two cases are only differed by a small factor and thus the bandwidth of negative permittivity is not significantly modified when the local field effect is taken into consideration. Similar proof can also be applied to analysis of the bandwidth of negative permeability. In conclusion, the local field effect would not substantially modify the bandwidth of negative refraction.

\section{Appendix F: Poynting Vector and the Wave Vector Surface}

In this section, we follow the treatment in Ref. 33] to prove that Poynting vector is parallel or anti-parallel to the normal of the wave vector surface. Let $\vec{n}$ be a point on the wave vector surface

$$
f\left(n_{x}, n_{y}, n_{z}\right)=0
$$

and $\delta \vec{n}$ be such a small change to $\vec{n}$ on the wave-vector surface that $\delta \vec{n}$ can be viewed as a vector in the tangent plane at that point, i.e., $\delta \vec{n} \cdot \partial f / \partial \vec{n}=0$. Here, $\partial f / \partial \vec{n}$ is the normal of the wave vector surface at the point $\vec{n}$.

According to the Maxwell's equations, we have

$$
\begin{aligned}
\vec{k} \times \vec{E} & =\omega \mu_{0} \overleftrightarrow{\mu_{r}} \cdot \vec{H}, \\
\vec{k} \times \vec{H} & =-\omega \varepsilon_{0} \overleftrightarrow{\varepsilon_{r}} \cdot \vec{E}
\end{aligned}
$$

In combination with $\vec{k}=\omega \sqrt{\varepsilon_{0} \mu_{0}} \vec{n}$, these yield

$$
\begin{aligned}
\delta \vec{n} \times \vec{E}+\vec{n} \times \delta \vec{E} & =c \delta \vec{B}, \\
\delta \vec{n} \times \vec{H}+\vec{n} \times \delta \vec{H} & =-c \delta \vec{D} .
\end{aligned}
$$

By multiplying both sides of Eq. (F4) by $\vec{H}$, we have

$$
\begin{aligned}
c \vec{H} \cdot \delta \vec{B} & =\vec{H} \cdot(\delta \vec{n} \times \vec{E})+\vec{H} \cdot(\vec{n} \times \delta \vec{E}) \\
& =\vec{S} \cdot \delta \vec{n}-\delta \vec{E} \cdot(\vec{n} \times \vec{H}) \\
& =\vec{S} \cdot \delta \vec{n}+c \delta \vec{E} \cdot \vec{D} .
\end{aligned}
$$

Similarly, multiplying both sides of Eq. (F4) by $\vec{E}$ yields

$$
\begin{aligned}
-c \vec{E} \cdot \delta \vec{D} & =\vec{E} \cdot(\delta \vec{n} \times \vec{H})+\vec{E} \cdot(\vec{n} \times \delta \vec{H}) \\
& =-\vec{S} \cdot \delta \vec{n}-\delta \vec{H} \cdot(\vec{n} \times \vec{E}) \\
& =-\vec{S} \cdot \delta \vec{n}-c \delta \vec{H} \cdot \vec{B} .
\end{aligned}
$$

It follows from Eqs. (F6]F7) that

$$
\vec{S} \cdot \delta \vec{n}=\frac{1}{2} c[\vec{H} \cdot \delta \vec{B}-\delta \vec{H} \cdot \vec{B}+\vec{E} \cdot \delta \vec{D}-\delta \vec{E} \cdot \vec{D}]
$$

Because $\overleftrightarrow{\varepsilon_{r}}$ and $\overleftrightarrow{\mu_{r}}$ are independent on $\vec{n}[33]$,

$$
\begin{aligned}
\vec{H} \cdot \delta \vec{B} & =\vec{H} \cdot \overleftrightarrow{\mu_{r}} \cdot \delta \vec{H} \\
& =\sum_{i j} H_{i} \mu_{r}^{(i j)} \delta H_{j} .
\end{aligned}
$$

For symmetric $\overleftrightarrow{\mu_{r}}$ and $\overleftrightarrow{\varepsilon_{r}}$, i.e.

$$
\mu_{r}^{(i j)}=\mu_{r}^{(j i)}, \varepsilon_{r}^{(i j)}=\varepsilon_{r}^{(j i)},
$$

we have

$$
\begin{aligned}
\vec{H} \cdot \delta \vec{B} & =\sum_{i j} H_{i} \mu_{r}^{(j i)} \delta H_{j} \\
& =\delta \vec{H} \cdot \overleftrightarrow{\mu_{r}} \cdot \vec{H} \\
& =\delta \vec{H} \cdot \vec{B} .
\end{aligned}
$$

Similarly, we can also prove that

$$
\begin{aligned}
\vec{E} \cdot \delta \vec{D} & =\sum_{i j} E_{i} \varepsilon_{r}^{(i j)} \delta E_{j} \\
& =\sum_{i j} E_{i} \varepsilon_{r}^{(j i)} \delta E_{j} \\
& =\delta \vec{E} \cdot \vec{D} .
\end{aligned}
$$


Inserting Eqs. (F11F12) into Eq. (F8) yields

$$
\vec{S} \cdot \delta \vec{n}=0
$$

Equation (F13) indicates that the Poynting vector $\vec{S}$ is along the normal of the wave vector surface at given point $\vec{n}$.
[1] V. G. Veselago, Sov. Phys. Uspekhi. 10, 509 (1968).

[2] J. B. Pendry, Science 306, 1353 (2004).

[3] U. Leonhardt, Science 312, 1777 (2006).

[4] J. B. Pendry, D. Schurig, and D. R. Smith, Science 312, 1780 (2006).

[5] J. B. Pendry, Phys. Rev. Lett. 85, 3966 (2000).

[6] Y. Shen and Q. Ai, Sci. Rep. 6, 20336 (2016).

[7] J. B. Pendry, A. J. Holden, D. J. Robbins, and W. J. Stewart, IEEE Trans. Microw. Theory Tech. 47, 2075 (1999).

[8] D. R. Smith, W. J. Padilla, D. C. Vier, S. C. NematNasser, and S. Schultz, Phys. Rev. Lett. 84, 4184 (2000).

[9] W. J. Padilla, D. N. Basov, and D. R. Smith, Mater. Today 9, 28 (2006).

[10] C. W. Chang, M. Liu, S. Nam, S. Zhang, Y. Liu, G. Bartal, and X. Zhang, Phys. Rev. Lett. 105, 235501 (2010).

[11] R. C. McPhedran, I. V. Shadrivov, B. T. Kuhlmey, and Y. S. Kivshar, NPG Asia Mater. 3, 100 (2011).

[12] C. M. Soukoulis and M. Wegener, Nat. Photonics 5, 523 (2011).

[13] Y. F. Chen, P. Fischer, and F. W. Wise, Phys. Rev. Lett. 95, 067402 (2005).

[14] Q. Thommen and P. Mandel, Phys. Rev. Lett. 96, 053601 (2006).

[15] Y. Shen, H. Y. Ko, Q. Ai, S. M. Peng, and B. Y. Jin, J. Phys. Chem. C 118, 3766 (2014).

[16] P. P. Orth, R. Hennig, C. H. Keitel, and J. Evers, New J. Phys. 15, 013027 (2013).

[17] F. Brechtefeld, N. Lindlein, G. Leuchs, and U. Peschel, Ring-Shaped Molecules as Split Ring Resonators of a Molecular Metamaterial, in Photonic Metamaterials: From Random to Periodic, Technical Dgest (CD) (Optical Society of America, 2006), paper WD21.

[18] E. Heilbronner, Tetrahedron Lett. 5, 1923 (1964).

[19] N. Zhao, H. Dong, S. Yang, and C. P. Sun, Phys. Rev. B 79, 125440 (2009).

[20] D. Ajami, O. Oeckler, A. Simon, and R. Herges, Nature 426, 819 (2003).

[21] T. Yoneda, Y. M. Sung, J. M. Lim, D. Kim, and A. Osuka, Angew. Chem. 126, 13385 (2014).

[22] V. Balzani, A. Credi, and M. Venturi, Molecular Devices and Machines: Concepts and Perspectives for the Nanoworld (VCH-Wiley, Weinheim, 2008).

[23] N. Byers and C. N. Yang, Phys. Rev. Lett. 7, 46 (1961).

[24] Z. L. Guo, Z. R. Gong, H. Dong, and C. P. Sun, Phys.
Rev. B 80, 195310 (2009).

[25] D. M. Walba, T. C. Homan, R. M. Richards, and R. C. Haltiwanger, New J. Chem. 17, 661 (1993).

[26] L. Salem, The Molecular Orbital Theory of Conjugated Systems (Benjamin, Reading, MA, 1972).

[27] A. Ceulemans, L. F. Chibotaru, and P. W. Fowler, Phys. Rev. Lett. 80, 1861 (1998).

[28] J. D. Jackson, Classical Electrodynamics 3rd Ed., (John Wiley, United States, 1999).

[29] R. Marqués, F. Martín, and M. Sorolla, Metamaterials with Negative Parameters: Theory, Design and $\mathrm{Mi}$ crowave Applications (John Wiley, New Jersey, 2008).

[30] R. Kubo, M. Toda, and N. Hashitsume, Statistical Physics II Nonequlibirum Statistical Mechanics (Springer-Verlag, Berlin Heidelberg, 1985).

[31] H. H. Greenwood, Computing Methods in Quantum Organic Chemistry (Wiley-Interscience, Germany, 1972).

[32] R. J. Silbey, R. A. Alberty, and M. G. Bawendi, Physical Chemistry, 4th Ed. (John Wiley\&Sons, Hoboken, 2004).

[33] L. D. Landau, E. M. Lifshitz, and L. P. Pitaevskii, Electrodynamics of Continuous Media 2nd Ed., Chapter 11, (Butterworth Heinmann, Oxford, 1995).

[34] N. H. Shen, Q. Wang, J. Chen, Y. X. Fan, J. P. Ding, H. T. Wang, Y. Tian, and N. B. Ming, Phys. Rev. B 72, 153104 (2005).

[35] L. B. Hu and S. T. Chui, Phys. Rev. B 66, 085108 (2002).

[36] P. W. Tao, L. S. Hua, and Q. Z. Liang, Chin. Phys. Lett. 29, 034102 (2012).

[37] R. A. Depine and A. Lakhtakia, Microw. Opt. Techn. Lett. 41, 315 (2004).

[38] M. W. McCall, A. Lakhtakia, and W. S. Weiglhofer, Eur. J. Phys. 23, 353 (2002).

[39] S. Tokuji, J.-Y. Shin, K. S. Kim, J. M. Lim, K. Youfu, S. Saito, D. Kim, and A. Osuka, J. Am. Chem. Soc. 131, 7240 (2009).

[40] Z. S. Yoon, A. Osuka, and D. Kim, Nat. Chem. 1, 113 (2009)

[41] E. Estrada and Y. Simó-Manso, Chem. Phys. Lett. 548, 80 (2012).

[42] S. Mukamel, Principles of Nonlinear Optical Spectroscopy (Oxford University Press, New York, 1995).

[43] L. Hawkea, G. Kalosakasa, and C. Simserides, Mol. Phys. 107, 1755 (2009). 